\title{
Focused Ultrasound of the Heart and Lungs in Patients With Respiratory Failure: A Randomized Controlled Multicenter Trial.
}

\author{
Minna Riishede ( $\triangle$ Minna.Riishede@rsyd.dk) \\ Syddansk Universitet https://orcid.org/0000-0002-5785-3317 \\ Annmarie T. Lassen \\ University of Southern Denmark, Odense University Hospital \\ Gunnar Baatrup \\ University of Southern Denmark, Odense University Hospital \\ Pia Iben Pietersen \\ University of Southern Denmark, Odense University Hospital \\ Niels Jacobsen \\ University of Southern Denmark, Odense University Hospital \\ Klaus Nielsen Jeschke \\ Hvidovre Hospital \\ Christian Borbjerg Laursen \\ University of Southern Denmark, Odense University Hospital
}

\section{Original research}

Keywords: Ultrasound, point of care ultrasonography, emergency department, respiratory disease, diagnostic accuracy, high acuity.

Posted Date: September 17th, 2020

DOI: https://doi.org/10.21203/rs.3.rs-75412/v1

License: (c) (i) This work is licensed under a Creative Commons Attribution 4.0 International License. Read Full License

Version of Record: A version of this preprint was published at Scandinavian Journal of Trauma, Resuscitation and Emergency Medicine on April 26th, 2021. See the published version at https://doi.org/10.1186/s13049-021-00872-8. 


\section{Abstract}

Background Ultrasound is a focus oriented tool for differentiating among cardiopulmonary diseases. Its value in the hands of emergency physicians, with various ultrasound experience, remains uncertain. We tested the hypothesis that, in emergency department patients with signs of respiratory failure, a focused cardiopulmonary ultrasound along with standard clinical examination, performed by emergency physicians with various ultrasound experience would increase the proportion of patients with correct diagnoses at 4 hours after admission compared to standard clinical examination alone.

Methods In this prospective multicenter superiority trial in Danish emergency departments we randomly assigned patients presenting with acute signs of respiratory failure to intervention or control in a 1:1 ratio by block randomization. Patients received a focused cardiopulmonary ultrasound examination within 4 hours from admission. Ultrasound results were unblinded for the treating emergency physician in the intervention group. Final diagnoses and treatment were determined by blinded review of the medical record after the patients' discharge.

Results From October 9, 2015 to April 5, 2017, we randomized 218 patients and included 211 in the final analyses. At 4 hours we found; no change in the proportion of patients with correct presumptive diagnoses; intervention $79 \cdot 25 \%(95 \% \mathrm{Cl}$ 70-3-86.0), control $77 \cdot 1 \%(95 \% \mathrm{Cl} 68 \cdot 0-84 \cdot 3)$, an increased proportion of correct treatment prescribed; intervention $79 \cdot 3 \%$ (95\% Cl 70.3-86.0), control $65 \cdot 7 \%(95 \% \mathrm{Cl} 56 \cdot 0-74 \cdot 3)$ and of patients who spent less than 1 day in hospital; intervention $n=42(39 \cdot 6 \% ; 25 \cdot 838 \cdot 4)$, control $n=25(23 \cdot 8 \% ; 16 \cdot 5-33 \cdot 0)$. No adverse events were reported.

Conclusions Focused cardiopulmonary ultrasound added to standard clinical examination in patients with signs of respiratory failure had no impact on the diagnostic accuracy, but significantly increased the proportion of correct treatment prescribed and the proportion who spent less than 1 day in hospital.

Trial registration https://clinicaltrials.gov/, number NCT 02550184.

\section{Background}

Patients admitted with acute signs of respiratory failure are a challenge for emergency physicians and among the most common indications for admission to the emergency department (ED) among adults. ${ }^{1,2}$

Symptoms are caused by a variety of respiratory and circulatory diseases of which some of the most common are exacerbation in chronic lung disease, pneumonia, pulmonary embolism and congestive heart failure. ${ }^{1,3} \mathrm{~A}$ fast and correct diagnosis can be crucial as vital signs are often close to normal in those potentially life-threatening diagnoses with a substantial risk of ICU admission. ${ }^{3}$ Moreover, these patients are among those with the highest over-all 30 -day mortality. ${ }^{4}$

Over the past decade focused ultrasound (F-us) examination in the ED has gained its place in the armamentarium of diagnostic tools. ${ }^{5,6}$

F-us of the lungs has proven to be a helpful tool in the assessment of patients with suspected respiratory diseases and has proven to be superior to chest $\mathrm{x}$-ray in the diagnostic assessment of various pulmonary diseases. ${ }^{7-9}$

Combined F-us of the heart and lungs has significantly improved the diagnostic accuracy of various cardiopulmonary diseases (e.g., pneumonia, pulmonary embolism, or edema) and identified life threatening diseases missed at primary clinical examination. ${ }^{10-12}$

Studies have demonstrated that novice sonographers can find pathology with accuracy comparable to expert sonographers and reference standards with only little training. ${ }^{13,14}$ 
The amount of diagnostic accuracy studies is vast and we are short of studies of the F-us' impact on patient related outcomes. Results from diagnostic accuracy studies are rarely directly translational to clinical practice hence we need randomized pragmatic studies to investigate the potential impact of F-us in daily clinical practice.

We investigated the hypothesis that adding a cardiopulmonary F-us to standard clinical examination of patients admitted to the ED with acute signs of respiratory failure could increase the proportion of patients with a correct presumptive diagnosis at 4 hours $(4 \mathrm{~h})$ after admission when compared to standard clinical examination alone. ${ }^{15}$

\section{Methods}

\section{Study design}

This prospective randomized semi-blinded, multicenter, superiority trial with a parallel group design and allocation ratio of 1:1 was undertaken in 10 of the 21 EDs in Danish community Hospitals. Three of these EDs were located at university hospitals and seven in secondary or tertiary hospitals. Patients were enrolled from October 9, 2015 to April 5, 2017.

Acute admissions to Danish EDs are to public hospitals and established by the general practitioner or by emergency call. Exceptions are patients suspected of having heart disease who are admitted directly to the cardiology department.

A presumptive diagnosis at admission and at $4 \mathrm{~h}$ as well as a treatment plan must ideally be journalized by the EP (emergency physician) within $4 \mathrm{~h}$ from the patient's admission to the ED.

\section{Inclusion and exclusion}

We screened patients admitted to the ED for inclusion. Patients $\geq 18$ years with a primary sign or symptom of respiratory failure of; cough, dyspnea, chest pain, respiratory frequency $>20$ or peripheral oxygen saturation $<95 \%$ or any combination of these were included upon written informed consent. Exclusion criteria were inability to give written informed consent, Fus of the lungs or heart already performed by others than the investigator in relation to the primary examination, or inability to randomize or perform the F-us within $4 \mathrm{~h}$ from the patient's admission to the ED.

\section{Randomization and masking}

Randomization numbers were created by a block randomization database (REDCap, OPEN) using permuted blocks of random numbers to ensure equal numbers of patients in both trial arms at each center. ${ }^{16}$ The allocation sequence of randomization numbers was generated by a data manager from REDCap OPEN and the project manager, MR, using an online random number service. Randomization numbers were paired with the REDCap OPEN database developed for this trial by MR.

The investigators screened the patients for inclusion at alternating shifts including day and night shifts on all weekdays. Once a patient signed the informed consent, the investigator registered the patient in the trial database that randomly allocated the patient and assigned the patient a unique computerized randomization number. Patients were aware of their group assignment.

The investigator then noted the basic clinical values upon the patient's admission and performed the F-us unaware of the primary presumptive diagnoses. All F-us results were entered in the project database.

F-us results from patients in the control group remained blinded to the EP, whereas once the investigator had received the primary presumptive diagnoses, he unblinded the intervention groups F-us results to the EP both orally and in writing on a paper record marked with the patients ID number and stored it in an accessible briefcase in the ED. The EP was then free to re-evaluate his presumptive diagnoses and treatment according to the ED's clinical guidelines. The EP was instructed to 
leave all information of randomization and F-us results out of the medical records to ensure blinding of the medical record audit.

In the medical record the investigator only noted the projects identification number and localization of the paper records containing the F-us results.

\section{Procedures}

In all patients admitted to the ED the EP assessed the primary presumptive diagnoses using standard methods of diagnostic examination (e.g., clinical examination, blood samples, ECG, chest x-ray) as soon as possible after the patient's arrival.

Standard diagnostic tests were available within 4h. Supplementary imaging examinations such as computerized tomography (CT), ultrasonography and echocardiography performed by specialists were available if necessary.

The investigator performed the F-us blinded to the EP in both groups. Subsequently, the EP announced his primary presumptive diagnoses to the investigator who then unblinded the F-us findings in the intervention group.

The F-us was performed within 4h from the patients' admission to the ED and consisted of F-us of the heart and lungs. The F-us protocol was defined as follows:

The F-us of the lungs was a modification of the ultrasound protocol used by Laursen et al. originally modified from the principles of lung ultrasound by Volpicelli and Lichtenstein. ${ }^{12,17,18}$ It was performed as follows: The anterior and lateral part of thorax was divided into a superior and inferior quadrant. Each quadrant represented a zone in which the probe was placed centrally to create a transverse picture of the costae and pleurae. We looked for pleural effusion, interstitial syndrome/pulmonary edema and pneumothorax.

The focused cardiac ultrasound was performed according to the principles described in the international evidence based guideline. ${ }^{19}$ The views used were the 4-chamber picture of the heart achieved either from a sub-xiphoid or an apical window. We looked for pericardial effusion, altered left ventricular ejection fraction and right ventricular overload.

There were no regulations in the choice of ultrasound machines or probes used for inclusion as long as the F-us was performed with an image quality deemed sufficient for evaluation by the operator.

All F-us examinations were performed by investigators who were either specialists or in specialist training and who received patients in the ED on a regular basis. All investigators used F-us on a daily basis in their clinical practice but had varying degrees of F-us experience. Prior to become an investigator they all received an educational program regarding data collection and F-us examination, which was composed by MR and consisted of an e-learning presentation with instructional videos. ${ }^{20}$ Then, MR made a $4 \mathrm{~h}$ on site presentation comprising the project, the collection and registration of data and an introduction to the project's F-us protocol. Hereafter, MR evaluated each investigator's F-us skills and handling of the project database by hands on and by multiple choice questionnaire tests to ensure that the investigators were familiar with their investigator tasks prior to initiation of inclusion. During the period of inclusion, investigators could take daily contact to the project manager for questions.

The diagnostic criteria for the F-us examination are provided in Appendix 1.

\section{Diagnostic examinations}

The primary presumptive diagnoses were registered in the medical record. The EP was free to reassess the presumptive diagnoses, diagnostic tests, and treatment. 
The $4 \mathrm{~h}$ presumptive diagnosis was the last registered clinical examination made by the EP within $4 \mathrm{~h}$ from the patients' admission to the ED and was assessed by blinded audit of the medical record after the patients discharge from hospital. New diagnostic tests and treatments could be prescribed after the $4 \mathrm{~h}$ examination but would then be part of the final diagnosis.

The final diagnosis was assessed by blinded audit of the medical record and included electronic journal data (e.g. clinical, microbiological and biochemical data, and imaging results) and was performed after the patients' discharge from hospital by two auditors, who independently of each other, set the final diagnosis. In case of discrepancy a third auditor set the final diagnosis. A predefined audit protocol with diagnostic criteria of the most common diagnoses was used (Appendix 2).

\section{Outcomes}

The primary outcome was to assess the percentage of patients with a correct presumptive diagnosis at $4 \mathrm{~h}$ after the patient 's admission to the ED. The $4 \mathrm{~h}$ cut off was set because various Danish hospitals request that examination, presumptive diagnoses and plan for further treatment are assessed within $4 \mathrm{~h}$ from admission to the ED. ${ }^{21}$

The secondary outcomes were as follows:

1. Diagnostic accuracy of the primary presumptive diagnoses made upon arrival.

2. The proportion of patients receiving a correct primary presumptive diagnosis made upon arrival.

3. The proportion of patients who, within $4 \mathrm{~h}$ after admission to the ED, is given the correct treatment.

4. Time spent in the ED (hours).

5. Time spent in hospital (days).

6. The proportion of patients being transferred from the ED to the intensive care unit.

7. The proportion of patients being transferred from the ED to a hospital ward.

8. The proportion of patients being discharged from the ED and directly to their home.

9. The proportion of patients being readmitted to hospital within 30 days from discharge.

10. In-hospital mortality.

11. 30-day mortality.

As a negative F-us can be found in various pulmonary diseases (e.g. chronic obstructive pulmonary disease (COPD), asthma) we could not compare F-us findings directly with final diagnoses and were omitted from the secondary endpoint 'The proportion of F-us examinations with a correct diagnostic examination' as written in the published protocol. ${ }^{15}$

\section{Unblinding}

For safety and ethical reasons the F-us findings in the control group were unblinded to the EP in charge of the patient if the F-us raised suspicion of a life-threatening condition (e.g. pulmonary edema, pneumothorax, pericardial effusion, or heart failure). ${ }^{15}$

\section{Statistical analysis}

The sample size estimate was based on the results from a previous similar trial where about $65 \%$ of the patients had a correct diagnosis at $4 \mathrm{~h}$ after admission to the ED when F-us was not used. ${ }^{11} \mathrm{~A}$ clinically significant improvement of the diagnosis by using F-us in a multicenter trial was set to be $15 \%$.

To detect a $15 \%$ increase in the number of correct tentative diagnoses, from $65 \%$ in the control group to $80 \%$ in the intervention group, with an $80 \%$ chance for detection, a level of significance of $5 \%$ and with an estimated drop out of $6 \%$ 
we had to include 288 patients, 144 patients in each group (intervention/control). Sample size calculations were made with the online database for clinical trials; Sealed Envelope. ${ }^{22}$

We used the intention-to-treat method as main comparative analysis on all participants. Descriptive statistics were handled as follows: Categorical data by number and percentages of patients with $95 \%$ confidence intervals (Cl), continuous data by number of patients $(n)$, mean, standard deviation, median, minimum and maximum.

Missing data in the baseline characteristics were handled as simple imputation when represented as dichotomous data. Other missing data were evaluated to be missing at random and was handled by multiple imputations using the Markov Chain Monte-Carlo method including auxiliary variables in the model. We plotted the mean value of the imputed variables in the spot of missing data. The rule of three was used to find $95 \% \mathrm{Cl}$ in categories without events. Categorical endpoints were summarized by numbers and percentages with $95 \%$ confidence intervals. ${ }^{23}$

We used the $\mathrm{Chi}^{2}$ test and the Fischer exact test for comparison of proportions expressed as percentages and for the comparison of continuous endpoints we used the Student $t$ test (means) and the Mann Whitney test (medians). A twosided significance level of $5 \%$ was applied to all tests.

We used the diagnoses from blinded audit as reference test for determining the diagnostic accuracy of the presumptive diagnoses established at admission and at $4 \mathrm{~h}$ and their $95 \%$ confidence interval based at binominal distribution. To assess the interrater reliability of the final diagnoses we used the Cohens kappa coefficient. Data analyses were conducted using STATA Release V. 15.0 (Stata Corp) including professional statistical advice. To assess the time a patient spent in the ED or hospital and the mortality we withdrew data from the Danish National Patient Register (LPR) administered by the Danish Health Data Authority. No data monitoring committee oversaw the trial.

\section{Role of the funding source}

Funders of this trial had no role in the trial design, data collection, data analysis, data interpretation or writing of the report. The corresponding author had full access to all the trial data and had final responsibility for the decision to submit for publication.

\section{Results}

We included 220 patients from October 9, 2015 to April 5, 2017 in 10 hospitals counting three university hospitals and seven tertiary hospitals with a total of 21 investigators. The trial was ended before we reached the predefined sample size as investigators travelled to other departments and the inclusion stagnated. We initially excluded 9 patients: two patient contacts due to double inclusions (their latest inclusions were excluded), one patient who withdrew informed consent, one patient who had no randomization or F-us within $4 \mathrm{~h}$ from admission and seven patients due to loss to follow up.

For the intention to treat analyses 211 patients (96\%) were randomized: intervention $n=106(50 \%)$ and control $n=105$ (50\%). We then excluded 10 patients due to non-blinding of the F-us results and ended with 201 patients (91\%) in the per protocol analyses; intervention $n=102$ (51\%), control $n=99$ (49\%) (Trial profile Fig. 1).

Figure 1 Trial profile (appended)

There are no deviations of the F-us examination in either group due to adverse events or complications related to the trial. For patient baseline characteristics see Table 1. For ultrasound findings see Appendix 3. 
Baseline characteristics of the intention to treat population.

\begin{tabular}{|lll|}
\hline & Intervention group $(\mathbf{n}=106)$ & Control group $(\mathbf{n}=\mathbf{1 0 5})$ \\
\hline Age (years; median (IQR) & $68(51-79)$ & $69(62-79)$ \\
\hline Sex & $62(58 \%)$ & $46(44 \%)$ \\
Male & $44(42 \%)$ & $59(56 \%)$ \\
\hline Smoking status & & \\
Never smoked & $30(28 \%)$ & $25(24 \%)$ \\
Current smoker & $27(25 \%)$ & $12(11 \%)$ \\
Previous smoker & $24(23 \%)$ & $37(35 \%)$ \\
Unknown status & $25(24 \%)$ & $31(30 \%)$ \\
\hline Medical history & & $7(7 \%)$ \\
Apoplexy & $9(8 \%)$ & $16(15 \%)$ \\
Coronary artery disease & $17(16 \%)$ & $14(13 \%)$ \\
Heart failure & $13(12 \%)$ & $22(21 \%)$ \\
Arterial hypertension & $24(23 \%)$ & $5(5 \%)$ \\
Thromboembolic disease & $11(10 \%)$ & $32(30 \%)$ \\
Chronic obstructive pulmonary disease & $33(31 \%)$ & $6(6 \%)$ \\
Asthma & $6(6 \%)$ & $9(9 \%)$ \\
Other pulmonary or pleural lung disease & $11(10 \%)$ & $17(16 \%)$ \\
Diabetes mellitus & $8(8 \%)$ & $8(8 \%)$ \\
Chronic kidney disease & $8(8 \%)$ & $36(34 \%)$ \\
Other medical disease & $28(26 \%)$ & $4(4 \%)$ \\
Psychiatric disorder & $7(7 \%)$ & \\
\hline
\end{tabular}

Data are number (n), (\%),mean (SD) or a median (IQR), unless otherwise indicated.

Data are not available for all randomized patients. Missing data are handled by multiple imputation for continuous data and simple imputation when binominary.

${ }^{a}$ We found GCS 9 in one patient admitted with exacerbation in terminal COPD. Pt was immediately treated with NIV with effect, replied relevant on questions and signed informed consent. The remaining of the included patients had GCS from $14-15$.

$\mathrm{b}$ The severity score is made according to the Danish Emergency Process Triage (DEPT) criteria used for patients with acute illness. The severity score is assessed by measuring the patients' vital parameters (e.g. BP, HR, GCS). 'I (Red)' is the most severe condition. 


\begin{tabular}{|lll|}
\hline & Intervention group $(\mathbf{n}=106)$ & Control group $(\mathbf{n}=105)$ \\
\hline Medication at admission & $28(26 \%)$ & $23(22 \%)$ \\
B-blockers & $38(36 \%)$ & $49(47 \%)$ \\
Nitrates & $9(8 \%)$ & $14(13 \%)$ \\
Angiotensin-converting-enzyme inhibitor & $26(25 \%)$ & $38(36 \%)$ \\
or angiotensin-receptor blocker & $4(4 \%)$ & $5(5 \%)$ \\
Digoxin & $16(15 \%)$ & $21(20 \%)$ \\
Calcium-channel blockers & $16(15 \%)$ & $18(17 \%)$ \\
Aspirin & $39(37 \%)$ & $45(43 \%)$ \\
Inhaled bronchodilators & $21(20 \%)$ & $31(30 \%)$ \\
Inhaled corticosteroids & $10(9 \%)$ & $17(16 \%)$ \\
Oral corticosteroids & $18(17 \%)$ & $17(16 \%)$ \\
Antibiotics & $29(27 \%)$ & $36(34 \%)$ \\
Anticoagulants & $5(5 \%)$ & $2(2 \%)$ \\
Immunosuppressive medication & $19(18 \%)$ & $24(23 \%)$ \\
Other medication & & \\
\hline
\end{tabular}

Data are number (n), (\%), mean (SD) or a median (IQR), unless otherwise indicated.

Data are not available for all randomized patients. Missing data are handled by multiple imputation for continuous data and simple imputation when binominary.

aWe found GCS 9 in one patient admitted with exacerbation in terminal COPD. Pt was immediately treated with NIV with effect, replied relevant on questions and signed informed consent. The remaining of the included patients had GCS from 14-15.

$\mathrm{b}$ The severity score is made according to the Danish Emergency Process Triage (DEPT) criteria used for patients with acute illness. The severity score is assessed by measuring the patients' vital parameters (e.g. BP, HR, GCS). 'I (Red)' is the most severe condition. 


\begin{tabular}{|c|c|c|}
\hline & Intervention group $(n=106)$ & Control group $(n=105)$ \\
\hline Vital signs at admission mean, (min-max) & $21(12-44)$ & $22(12-40)$ \\
\hline Respiratory rate (breaths per min) & $94(55-100)$ & $95(74-100)$ \\
\hline Saturation (\%) & $23(22 \%)$ & $29(28 \%)$ \\
\hline - of these patients $n$ received oxygen supply ( $/ / \mathrm{min})$ & $15(65 \%)$ & $24(83 \%)$ \\
\hline o $1-3 \mathrm{l} / \mathrm{min} \mathrm{n}(\%)$ & $8(35 \%)$ & $4(14 \%)$ \\
\hline o $4-6 \mathrm{l} / \mathrm{min} \mathrm{n}(\%)$ & 0 & $1(3 \%)$ \\
\hline $0>6 \mathrm{l} / \mathrm{min} \mathrm{n}(\%)$ & $137(80-224)$ & $137(94-212)$ \\
\hline Systolic blood pressure $(\mathrm{mmHg})$ & $78(32-123)$ & $78(47-111)$ \\
\hline Diastolic blood pressure $(\mathrm{mmHg})$ & $90(52-150)$ & $86(40-144)$ \\
\hline Heart rate (beats per minute) & $37.1(35.2-39.5)$ & $37.2(35.0-39.8)$ \\
\hline Temperature $\left({ }^{\circ} \mathrm{C}\right)$ & $6.8(4.1-19.9)$ & $6.6(1.0-22.4)$ \\
\hline
\end{tabular}

Data are number (n), (\%), mean (SD) or a median (IQR), unless otherwise indicated.

Data are not available for all randomized patients. Missing data are handled by multiple imputation for continuous data and simple imputation when binominary.

aWe found GCS 9 in one patient admitted with exacerbation in terminal COPD. Pt was immediately treated with NIV with effect, replied relevant on questions and signed informed consent. The remaining of the included patients had GCS from 14-15.

$\mathrm{b}$ The severity score is made according to the Danish Emergency Process Triage (DEPT) criteria used for patients with acute illness. The severity score is assessed by measuring the patients' vital parameters (e.g. BP, HR, GCS). 'I (Red)' is the most severe condition. 


\begin{tabular}{|c|c|c|}
\hline & Intervention group $(n=106)$ & Control group $(n=105)$ \\
\hline Patients' signs and symptoms upon admission & $33(31 \%)$ & $43(41 \%)$ \\
\hline Cough & $90(85 \%)$ & $85(81 \%)$ \\
\hline Dyspnoea & $28(26 \%)$ & $28(27 \%)$ \\
\hline Chest pain & $33(30 \%)$ & $30(29 \%)$ \\
\hline Respiration rate $>20$ breaths per min & $30(28 \%)$ & $24(23 \%)$ \\
\hline Peripheral saturation $<95 \%$ & 0 & 0 \\
\hline \multicolumn{3}{|l|}{ None of the above } \\
\hline Ultrasound examination already performed in ambulance & 0 & 0 \\
\hline Patients severity score upon admission ${ }^{\mathrm{b}}$ & $5(5 \%)$ & $1(1 \%)$ \\
\hline - I (Red) & $29(27 \%)$ & $36(34 \%)$ \\
\hline - II (Orange) & $49(46 \%)$ & $42(40 \%)$ \\
\hline - III (Yellow) & $23(22 \%)$ & $27(26 \%)$ \\
\hline \multicolumn{3}{|l|}{ - IV (Green) } \\
\hline \multicolumn{3}{|c|}{ Data are number $(\mathrm{n}),(\%)$,mean (SD) or a median (IQR), unless otherwise indicated. } \\
\hline \multicolumn{3}{|c|}{$\begin{array}{l}\text { Data are not available for all randomized patients. Missing data are handled by multiple imputation for continuous } \\
\text { data and simple imputation when binominary. }\end{array}$} \\
\hline \multicolumn{3}{|c|}{$\begin{array}{l}\text { aWe found GCS } 9 \text { in one patient admitted with exacerbation in terminal COPD. Pt was immediately treated with NIV } \\
\text { with effect, replied relevant on questions and signed informed consent. The remaining of the included patients had } \\
\text { GCS from 14-15. }\end{array}$} \\
\hline \multicolumn{3}{|c|}{$\begin{array}{l}\text { b The severity score is made according to the Danish Emergency Process Triage (DEPT) criteria used for patients with } \\
\text { acute illness. The severity score is assessed by measuring the patients' vital parameters (e.g. BP, HR, GCS). "I (Red) ' is } \\
\text { the most severe condition. }\end{array}$} \\
\hline
\end{tabular}

Final diagnoses were assessed by the two auditors with an overall agreement of $93 \cdot 18 \%$ (kappa $0 \cdot 58$ ) ranging from $81 \cdot 04-100 \%$ (kappa - 0.02-1.0) within the specific diagnoses. A detailed description of the interrater reliability of the audit is provided in Appendix 4. The most common final diagnoses in the intervention and control group were; pneumonia (25\%/ $32 \%)$, exacerbation of COPD (25\%/27\%) and systolic heart failure (16\%/21\%). The proportion of correctly assessed diagnoses at $4 \mathrm{~h}$ in the intervention group is increased compared to the control group for the diagnoses of exacerbation of COPD (89\%/75\%), pulmonary edema (53\%/33\%) and para-pneumonic effusion (77\%/38\%) but with overlapping Cls. The proportion of correctly diagnosed pneumonia is equal among the groups (88\%/88\%) (Table 2,3$)$. 
Table 2

Final diagnoses and the proportion of correctly assessed diagnoses at 4 hours from admission.

\begin{tabular}{|c|c|c|c|c|}
\hline & \multicolumn{2}{|c|}{$\begin{array}{l}\text { Diagnoses in the intervention group }(n= \\
\text { 106) }\end{array}$} & \multicolumn{2}{|c|}{ Diagnoses in the control group $(n=105)$} \\
\hline & $\begin{array}{l}\text { Final } \\
\text { diagnoses, }\end{array}$ & $\begin{array}{l}\text { Number of final diagnoses } \\
\text { found at } 4 \mathrm{~h}, \mathrm{n}(\%)\end{array}$ & $\begin{array}{l}\text { Final audit } \\
\text { diagnoses, } \mathrm{n}(\%)\end{array}$ & $\begin{array}{l}\text { Number of final diagnoses } \\
\text { found at } 4 \mathrm{~h}, \mathrm{n}(\%)\end{array}$ \\
\hline & $\mathrm{n}(\%)$ & & & \\
\hline \multicolumn{5}{|l|}{ Lungs } \\
\hline $\begin{array}{l}\text { Exacerbation of } \\
\text { COPD }\end{array}$ & $27(25 \%)$ & $24(89 \%)$ & $28(27 \%)$ & 21 (75\%) \\
\hline $\begin{array}{l}\text { Asthma with } \\
\text { exacerbation }\end{array}$ & $2(2 \%)$ & 0 & $3(3 \%)$ & $2(67 \%)$ \\
\hline $\begin{array}{l}\text { Exacerbation in } \\
\text { ILD }\end{array}$ & $3(3 \%)$ & $1(33 \%)$ & $5(5 \%)$ & 0 \\
\hline Pneumonia & $26(25 \%)$ & $23(88 \%)$ & $34(32 \%)$ & $30(88 \%)$ \\
\hline $\begin{array}{l}\text { Pulmonary } \\
\text { edema }\end{array}$ & $17(16 \%)$ & $9(53 \%)$ & $6(6 \%)$ & $2(33 \%)$ \\
\hline $\begin{array}{l}\text { Parapneumonic } \\
\text { effusion }\end{array}$ & $14(13 \%)$ & $10(77 \%)$ & $14(13 \%)$ & $5(38 \%)$ \\
\hline Empyema & $1(1 \%)$ & 0 & 0 & 0 \\
\hline $\begin{array}{l}\text { Pulmonary } \\
\text { embolism }\end{array}$ & 3 (3\%) & $3(100 \%)$ & $2(2 \%)$ & $2(100 \%)$ \\
\hline Pneumothorax & 0 & 0 & $1(1 \%)$ & $1(100 \%)$ \\
\hline \multicolumn{5}{|l|}{ Heart } \\
\hline $\begin{array}{l}\text { Systolic heart } \\
\text { failure }\end{array}$ & $17(16 \%)$ & $13(76 \%)$ & $22(21 \%)$ & $13(62 \%)$ \\
\hline $\begin{array}{l}\text { Non-systolic } \\
\text { heart failure }\end{array}$ & $5(5 \%)$ & $3(60 \%)$ & $1(1 \%)$ & $1(100 \%)$ \\
\hline $\begin{array}{l}\text { Acute myocardial } \\
\text { infarction }\end{array}$ & $1(1 \%)$ & 0 & $2(2 \%)$ & $1(50 \%)$ \\
\hline \multicolumn{5}{|l|}{ Miscellaneous } \\
\hline Anemia & $5(5 \%)$ & $1(20 \%)$ & $6(6 \%)$ & 0 \\
\hline Malignancy & $8(8 \%)$ & $2(25 \%)$ & $19(18 \%)$ & $5(26 \%)$ \\
\hline $\begin{array}{l}\text { No diagnostic } \\
\text { criteria met }\end{array}$ & $33(31 \%)$ & $19(58 \%)$ & $35(33 \%)$ & $21(60 \%)$ \\
\hline
\end{tabular}


Table 3

Diagnostic accuracy of emergency physicians' 4 hour presumptive diagnoses compared to final diagnoses (reference standard).

\begin{tabular}{|c|c|c|c|c|c|c|c|c|c|c|}
\hline \multirow[b]{2}{*}{ Diagnoses } & \multicolumn{2}{|c|}{$\begin{array}{l}\text { Final diagnosis } \\
\text { positive / } 4 \text { hour } \\
\text { positive }\end{array}$} & \multicolumn{2}{|c|}{$\begin{array}{l}\text { Sensitivity } \\
\%(95 \% \mathrm{Cl})\end{array}$} & \multicolumn{2}{|c|}{$\begin{array}{l}\text { Specificity } \\
\%(95 \% \mathrm{Cl})\end{array}$} & \multicolumn{2}{|c|}{$\begin{array}{l}\text { PPV } \\
\%(95 \% \mathrm{Cl})\end{array}$} & \multicolumn{2}{|c|}{$\begin{array}{l}\text { NPV } \\
\%(95 \% \mathrm{Cl})\end{array}$} \\
\hline & Interv. & Control & Interv. & Control & Interv. & Control & Interv. & Control & Interv. & Control \\
\hline $\begin{array}{l}\text { COPD with } \\
\text { exacerbation }\end{array}$ & $27 / 29$ & $28 / 24$ & $\begin{array}{l}89 \\
(71- \\
98)\end{array}$ & $\begin{array}{l}75 \\
(55- \\
89)\end{array}$ & $\begin{array}{l}94 \\
(86- \\
98)\end{array}$ & $\begin{array}{l}96 \\
(89- \\
99)\end{array}$ & $\begin{array}{l}83 \\
(64- \\
94)\end{array}$ & $\begin{array}{l}88 \\
(68- \\
97)\end{array}$ & $\begin{array}{l}96 \\
(89- \\
99)\end{array}$ & $\begin{array}{l}91 \\
(83- \\
97)\end{array}$ \\
\hline $\begin{array}{l}\text { Asthma with } \\
\text { exacerbation }\end{array}$ & $2 / 2$ & $3 / 3$ & $\begin{array}{l}0(0- \\
84)\end{array}$ & $\begin{array}{l}67(9- \\
99)\end{array}$ & $\begin{array}{l}98 \\
(93- \\
100)\end{array}$ & $\begin{array}{l}99 \\
(94- \\
100)\end{array}$ & $\begin{array}{l}0(0- \\
84)\end{array}$ & $\begin{array}{l}67(9- \\
99)\end{array}$ & $\begin{array}{l}98 \\
(93- \\
100)\end{array}$ & $\begin{array}{l}99 \\
(94- \\
100)\end{array}$ \\
\hline $\begin{array}{l}\text { Interstitial lung } \\
\text { disease }\end{array}$ & $3 / 1$ & $5 / 2$ & $\begin{array}{l}33 \\
(1- \\
91)\end{array}$ & $\begin{array}{l}0(0- \\
52)\end{array}$ & $\begin{array}{l}100 \\
(97- \\
100)\end{array}$ & $\begin{array}{l}98 \\
(93- \\
100)\end{array}$ & $\begin{array}{l}100(3- \\
100)\end{array}$ & $\begin{array}{l}0(0- \\
84)\end{array}$ & $\begin{array}{l}98 \\
(93- \\
100)\end{array}$ & $\begin{array}{l}95 \\
(88- \\
98)\end{array}$ \\
\hline Pneumonia & $26 / 34$ & $34 / 40$ & $\begin{array}{l}89 \\
(70- \\
98)\end{array}$ & $\begin{array}{l}88 \\
(73- \\
97)\end{array}$ & $\begin{array}{l}86 \\
(77- \\
93)\end{array}$ & $\begin{array}{l}86 \\
(76- \\
93)\end{array}$ & $\begin{array}{l}68 \\
(50- \\
83)\end{array}$ & $\begin{array}{l}75 \\
(59- \\
87)\end{array}$ & $\begin{array}{l}96 \\
(88- \\
99)\end{array}$ & $\begin{array}{l}94 \\
(85- \\
98)\end{array}$ \\
\hline $\begin{array}{l}\text { Pulmonary } \\
\text { edema }\end{array}$ & $17 / 10$ & $6 / 4$ & $\begin{array}{l}53 \\
(28- \\
77)\end{array}$ & $\begin{array}{l}33(4- \\
78)\end{array}$ & $\begin{array}{l}99 \\
(94- \\
100)\end{array}$ & $\begin{array}{l}98 \\
(93- \\
100)\end{array}$ & $\begin{array}{l}90 \\
(56- \\
100)\end{array}$ & $\begin{array}{l}50(7- \\
93)\end{array}$ & $\begin{array}{l}92 \\
(84- \\
96)\end{array}$ & $\begin{array}{l}96 \\
(90- \\
99)\end{array}$ \\
\hline $\begin{array}{l}\text { Para- } \\
\text { pneumonic } \\
\text { effusion }\end{array}$ & $14 / 14$ & $14 / 7$ & $\begin{array}{l}71 \\
(42- \\
92)\end{array}$ & $\begin{array}{l}36 \\
(13- \\
65)\end{array}$ & $\begin{array}{l}96 \\
(89- \\
99)\end{array}$ & $\begin{array}{l}98 \\
(92- \\
100)\end{array}$ & $\begin{array}{l}71 \\
(42- \\
92)\end{array}$ & $\begin{array}{l}71 \\
(29- \\
96)\end{array}$ & $\begin{array}{l}96 \\
(89- \\
99)\end{array}$ & $\begin{array}{l}91 \\
(83- \\
96)\end{array}$ \\
\hline $\begin{array}{l}\text { Pulmonary } \\
\text { empyema* }\end{array}$ & $1 / 0$ & $0 / 0$ & - & - & - & - & - & - & - & - \\
\hline $\begin{array}{l}\text { Pulmonary } \\
\text { emboli }\end{array}$ & $3 / 11$ & $2 / 7$ & $\begin{array}{l}100 \\
(29- \\
100)\end{array}$ & $\begin{array}{l}100 \\
(16- \\
100)\end{array}$ & $\begin{array}{l}92 \\
(85- \\
97)\end{array}$ & $\begin{array}{l}96 \\
(89- \\
98)\end{array}$ & $\begin{array}{l}27(6- \\
61)\end{array}$ & $\begin{array}{l}29(4- \\
71)\end{array}$ & $\begin{array}{l}100 \\
(96- \\
100)\end{array}$ & $\begin{array}{l}100 \\
(96- \\
100)\end{array}$ \\
\hline Pneumothorax* & $0 / 0$ & $1 / 3$ & - & $\begin{array}{l}100(3- \\
100)\end{array}$ & - & $\begin{array}{l}98 \\
(93- \\
100)\end{array}$ & - & $\begin{array}{l}33(1- \\
91)\end{array}$ & - & $\begin{array}{l}100 \\
(96- \\
100)\end{array}$ \\
\hline $\begin{array}{l}\text { Systolic heart } \\
\text { failure }\end{array}$ & $17 / 16$ & $22 / 18$ & $\begin{array}{l}77 \\
(50- \\
93)\end{array}$ & $\begin{array}{l}59 \\
(36- \\
79)\end{array}$ & $\begin{array}{l}97 \\
(91- \\
99)\end{array}$ & $\begin{array}{l}94 \\
(87- \\
98)\end{array}$ & $\begin{array}{l}81 \\
(54- \\
96)\end{array}$ & $\begin{array}{l}72 \\
(47- \\
90)\end{array}$ & $\begin{array}{l}95 \\
(89- \\
99)\end{array}$ & $\begin{array}{l}90 \\
(81- \\
95)\end{array}$ \\
\hline $\begin{array}{l}\text { Non-systolic } \\
\text { heart failure }\end{array}$ & $5 / 6$ & $1 / 2$ & $\begin{array}{l}60 \\
(15- \\
95)\end{array}$ & $\begin{array}{l}100(3- \\
100)\end{array}$ & $\begin{array}{l}97 \\
(92- \\
99)\end{array}$ & $\begin{array}{l}99 \\
(95- \\
100)\end{array}$ & $\begin{array}{l}50 \\
(12- \\
88)\end{array}$ & $\begin{array}{l}50(1- \\
99)\end{array}$ & $\begin{array}{l}98 \\
(93- \\
100)\end{array}$ & $\begin{array}{l}100 \\
(97- \\
100)\end{array}$ \\
\hline $\begin{array}{l}\text { Acute } \\
\text { myocardial } \\
\text { infarction }\end{array}$ & $1 / 2$ & $2 / 9$ & $\begin{array}{l}0(0- \\
98)\end{array}$ & $\begin{array}{l}50(1- \\
99)\end{array}$ & $\begin{array}{l}98 \\
(93- \\
100)\end{array}$ & $\begin{array}{l}92 \\
(85- \\
97)\end{array}$ & $\begin{array}{l}0(0- \\
84)\end{array}$ & $\begin{array}{l}11(0- \\
48)\end{array}$ & $\begin{array}{l}99 \\
(95- \\
100)\end{array}$ & $\begin{array}{l}99 \\
(94- \\
100)\end{array}$ \\
\hline Anemia & $5 / 1$ & $6 / 1$ & $\begin{array}{l}20 \\
(1- \\
72)\end{array}$ & $\begin{array}{l}0(0- \\
46)\end{array}$ & $\begin{array}{l}100 \\
(96- \\
100)\end{array}$ & $\begin{array}{l}99 \\
(95- \\
100)\end{array}$ & $\begin{array}{l}100(3- \\
100)\end{array}$ & $\begin{array}{l}0(0- \\
98)\end{array}$ & $\begin{array}{l}96 \\
(91- \\
99)\end{array}$ & $\begin{array}{l}94 \\
(88- \\
98)\end{array}$ \\
\hline Malignancy & $8 / 2$ & $19 / 7$ & $\begin{array}{l}25 \\
(3- \\
65)\end{array}$ & $\begin{array}{l}26(9- \\
51)\end{array}$ & $\begin{array}{l}94 \\
(96- \\
100)\end{array}$ & $\begin{array}{l}98 \\
(92- \\
100)\end{array}$ & $\begin{array}{l}100 \\
(16- \\
100)\end{array}$ & $\begin{array}{l}71 \\
(29- \\
96)\end{array}$ & $\begin{array}{l}94 \\
(88- \\
98)\end{array}$ & $\begin{array}{l}86 \\
(77- \\
92)\end{array}$ \\
\hline
\end{tabular}

Intervention $(\mathrm{n}=106)$, control $(\mathrm{n}=105)$. Intention to treat population. Abbreviation: Interv. (Intervention)

*Too few ratings to perform diagnostic accuracy calculations. 


\begin{tabular}{|c|c|c|c|c|c|c|c|c|c|c|}
\hline \multirow[b]{2}{*}{$\begin{array}{l}\text { Other } \\
\text { diagnoses }\end{array}$} & \multicolumn{2}{|c|}{$\begin{array}{l}\text { Final diagnosis } \\
\text { positive / } 4 \text { hour } \\
\text { positive }\end{array}$} & \multicolumn{2}{|c|}{$\begin{array}{l}\text { Sensitivity } \\
\%(95 \% \mathrm{Cl})\end{array}$} & \multicolumn{2}{|c|}{$\begin{array}{l}\text { Specificity } \\
\%(95 \% \mathrm{Cl})\end{array}$} & \multicolumn{2}{|c|}{$\begin{array}{l}\text { PPV } \\
\%(95 \% \mathrm{Cl})\end{array}$} & \multicolumn{2}{|c|}{$\begin{array}{l}\text { NPV } \\
\%(95 \% \mathrm{Cl})\end{array}$} \\
\hline & $33 / 29$ & $35 / 31$ & $\begin{array}{l}58 \\
(39- \\
75)\end{array}$ & $\begin{array}{l}60 \\
(42- \\
76)\end{array}$ & $\begin{array}{l}86 \\
(76- \\
93)\end{array}$ & $\begin{array}{l}86 \\
(75- \\
93)\end{array}$ & $\begin{array}{l}66(46- \\
82)\end{array}$ & $\begin{array}{l}68 \\
(49- \\
83)\end{array}$ & $\begin{array}{l}83(71- \\
90)\end{array}$ & $\begin{array}{l}81 \\
(70- \\
89)\end{array}$ \\
\hline
\end{tabular}

Intervention $(n=106)$, control $(n=105)$. Intention to treat population. Abbreviation: Interv. (Intervention)

*Too few ratings to perform diagnostic accuracy calculations.

No difference was found in the proportion of patients who received a correct presumptive diagnosis at arrival; intervention group, $n=65$ (61.32\%; 95\% Cl 51.59-70.23) versus control group, $n=65(61 \cdot 90 \% ; 95 \% \mathrm{Cl} 52 \cdot 12-70 \cdot 80),(p=0 \cdot 93)$ nor at $4 \mathrm{~h}$ after admission to the ED; intervention group, $n=84$ (79.25\%; 70.32-86.02) versus control group, $n=81$ (77.14\%; $68.00-84 \cdot 28)(p=0 \cdot 71)$. However, the proportion of patients who had a correct treatment prescribed at $4 \mathrm{~h}$ was significantly larger in the intervention group, $n=84$ (79.25\%; $95 \% \mathrm{Cl} 70 \cdot 32-86.02)$ than in the control group $n=69$ (65.71\%; $95 \% \mathrm{Cl} 55.99-74.27)(p=0.03)$ with an absolute increase of $13.5 \%$ (Table 4). No patients had F-us performed in the ambulance prior to admission to the ED. 
Table 4

Primary and secondary outcomes for the intention to treat population

\begin{tabular}{|c|c|c|c|c|c|}
\hline & $\begin{array}{l}\text { Intervention group (n } \\
=106) \\
\text { n (\%; } 95 \% \mathrm{Cl})\end{array}$ & $\begin{array}{l}\text { Control group }(n= \\
105) \\
n(\% ; 95 \% \mathrm{Cl})\end{array}$ & $\begin{array}{l}P \\
\text { value }\end{array}$ & $\begin{array}{l}\text { Absolute effect } \\
\%(95 \% \mathrm{Cl})\end{array}$ & $\begin{array}{l}\text { Relative effect } \\
(95 \% \mathrm{Cl})\end{array}$ \\
\hline \multicolumn{6}{|l|}{$\begin{array}{l}\text { PRIMARY } \\
\text { ENDPOINT }\end{array}$} \\
\hline $\begin{array}{l}4 \mathrm{~h} \text { after } \\
\text { admission to the } \\
\text { ED }\end{array}$ & 84 (79.3\%; 70.3-86.0) & 81 (77.1\%; 68.0-84.3) & 0.71 & $\begin{array}{l}2.1 \%(-9.0- \\
13.2)\end{array}$ & $1.03(0.89-1.18)$ \\
\hline \multicolumn{6}{|l|}{$\begin{array}{l}\text { Patients with } \\
\text { correct } \\
\text { presumptive } \\
\text { diagnosis }\end{array}$} \\
\hline \multicolumn{6}{|l|}{$\begin{array}{l}\text { SECONDARY } \\
\text { ENDPOINTS }\end{array}$} \\
\hline $\begin{array}{l}4 \mathrm{~h} \text { after } \\
\text { admission to the } \\
\text { ED }\end{array}$ & 84 (79.3\%; 70.3-86.0) & 69 (65.7\%; 56.0-74.3) & 0.03 & $\begin{array}{l}13.5 \%(1.6- \\
25.5)\end{array}$ & $\begin{array}{l}1.21(1.02- \\
1.43)\end{array}$ \\
\hline \multicolumn{6}{|l|}{$\begin{array}{l}\text { Appropriate } \\
\text { treatment ordered } \\
\text { a }\end{array}$} \\
\hline $\begin{array}{l}\text { After primary } \\
\text { assessment in the } \\
\text { ED }\end{array}$ & 65 (61.3\%; 51.6-70.2) & 65 (61.9\%; 52.1-70.8) & 0.93 & $\begin{array}{l}-0.5 \%(-13.7- \\
12.5)\end{array}$ & $0.99(0.80-1.23)$ \\
\hline $\begin{array}{l}\text { Patients with } \\
\text { correct } \\
\text { presumptive } \\
\text { diagnoses }\end{array}$ & & & & & \\
\hline
\end{tabular}

Abbreviations: $\mathrm{n}$ (number of patients). NIV (Non-invasive ventilation). CPAP (Continuous positive airway pressure). ED (emergency department). ICU (intensive care unit). $¥$ One-sided $97.5 \%$ confidence interval. aAccording to local treatment guidelines for the diseases in question. b Pleura-. thoraco-. cardio-centesis. c Performed by a radiologist or ultrasonographer certified in lung ultrasound. d Performed by radiologist or certified ultrasonographer. 


\begin{tabular}{|c|c|c|c|c|c|}
\hline & $\begin{array}{l}\text { Intervention group (n } \\
=106) \\
\text { n (\%; } 95 \% \mathrm{Cl})\end{array}$ & $\begin{array}{l}\text { Control group }(n= \\
105) \\
n(\% ; 95 \% \mathrm{Cl})\end{array}$ & $\begin{array}{l}P \\
\text { value }\end{array}$ & $\begin{array}{l}\text { Absolute effect } \\
\%(95 \% \mathrm{Cl})\end{array}$ & $\begin{array}{l}\text { Relative effect } \\
(95 \% \mathrm{Cl})\end{array}$ \\
\hline \multirow{3}{*}{$\begin{array}{l}\text { Specific treatment } \\
\text { prescribed within } \\
4 \mathrm{~h} \text { from } \\
\text { admission to the } \\
\text { ED }\end{array}$} & $33(31.1 \% ; 22.5-40.0)$ & 37 (35.2\%; 26. 2-45.2) & 0.53 & $\begin{array}{l}-4.1 \%(-16.8- \\
8.6)\end{array}$ & \multirow{2}{*}{$\begin{array}{l}0.88(0.60- \\
1.30)\end{array}$} \\
\hline & $3(2.80 \% ; 0.9-8.4)$ & $0\left(0 \% ; 0-3.5^{¥}\right)$ & 0.08 & $2.8 \%(-0.3-6.0)$ & \\
\hline & $0\left(0 \% ; 0-3.4^{¥}\right)$ & $0\left(0 \% ; 0-3.5^{¥}\right)$ & \multirow[b]{2}{*}{0.34} & +9 & - \\
\hline \multirow{2}{*}{$\begin{array}{l}\text { Oxygen } \\
\text { NIV/CPAP }\end{array}$} & $26(24.5 \% ; 16.5-34.0)$ & 20 (19.1\%; 12.0-27.9) & & \multirow{2}{*}{$\begin{array}{l}5.5 \%(-5.6- \\
16.6)\end{array}$} & \multirow{2}{*}{$\begin{array}{l}1.29(0.77- \\
2.16)\end{array}$} \\
\hline & $24(22.6 \% ; 14.9-31.9)$ & $21(20.0 \% ; 12.8-28.9)$ & 0.64 & & \\
\hline Respirator & $30(28.3 \% ; 19.9-38.2)$ & $38(36.2 \% ; 27.0-46.1)$ & 0.22 & $\begin{array}{l}2.6 \%(-8.4- \\
13.7)\end{array}$ & $\begin{array}{l}1.13(0.67- \\
1.90)\end{array}$ \\
\hline \multirow{2}{*}{$\begin{array}{l}\text { Bronchodilators } \\
\text { Systemic steroids }\end{array}$} & 15 (14.2\%; 7.7-22.0) & 25 (23.8\%; 16.0-33.1) & 0.07 & \multirow{2}{*}{$\begin{array}{l}-7.9 \%(-20.5- \\
4.7)\end{array}$} & \multirow{2}{*}{$\begin{array}{l}0.78(0.53- \\
1.16)\end{array}$} \\
\hline & $13(12.3 \% ; 7.0-20.8)$ & $12(11.4 \% ; 6.0-19.1)$ & 0.85 & & \\
\hline Antibiotics & $4(3.8 \% ; 1.1-9.7)$ & $3(2.9 \% ; 0.6-8.1)$ & 0.71 & $\begin{array}{l}-9.7 \%(-20.2- \\
0.8)\end{array}$ & $\begin{array}{l}0.59(0.33- \\
1.06)\end{array}$ \\
\hline \multirow{3}{*}{$\begin{array}{l}\text { Fluids i.v. } \\
\text { Diuretics } \\
\text { Antiarrythmics } \\
\text { Vasoconstrictors }\end{array}$} & $0\left(0 \% ; 0-3.4^{¥}\right)$ & $0\left(0 \% ; 0-3.5^{¥}\right)$ & & $0.8 \%(-7.9-9.6)$ & \multirow{2}{*}{$\begin{array}{l}1.07(0.51- \\
2.24)\end{array}$} \\
\hline & 10 (9.4\%; 4.8-17-3) & 13 (12.4\%; 6.8-20.2) & & $0.9 \%(-3.9-5.7)$ & \\
\hline & $3(2.8 \% ; 0.6-8.4)$ & $3(2.9 \% ; 0.6-8.1)$ & $\begin{array}{l}0.99 \\
0.59\end{array}$ & 0 & $\begin{array}{l}1.32(0.30- \\
5.76)\end{array}$ \\
\hline \multicolumn{6}{|l|}{ Anticoagulants } \\
\hline \multicolumn{6}{|l|}{$\begin{array}{l}\text { Therapeutic } \\
\text { centesis }^{b}\end{array}$} \\
\hline \multirow[t]{5}{*}{ Others } & & & & & \\
\hline & \multirow[t]{4}{*}{$8(7.6 \% ; 3.4-14.9)$} & \multirow[t]{4}{*}{$6(5.7 \% ; 2.1-12.0)$} & & $\begin{array}{l}-2.9 \%(-11.4- \\
5.5)\end{array}$ & \multirow{2}{*}{$\begin{array}{l}- \\
0.76(0.35- \\
1.66)\end{array}$} \\
\hline & & & & $-0.0 \%(-4.5-4.5)$ & \\
\hline & & & & $1.8(-4.9-8.5)$ & $\begin{array}{l}0.99(0.20- \\
4.80)\end{array}$ \\
\hline & & & & & $\begin{array}{l}1.32(0.47- \\
3.68)\end{array}$ \\
\hline
\end{tabular}

Abbreviations: $\mathrm{n}$ (number of patients). NIV (Non-invasive ventilation). CPAP (Continuous positive airway pressure). ED (emergency department). ICU (intensive care unit). $¥$ One-sided $97.5 \%$ confidence interval. aAccording to local treatment guidelines for the diseases in question. b Pleura-. thoraco-. cardio-centesis. c Performed by a radiologist or ultrasonographer certified in lung ultrasound. $d$ Performed by radiologist or certified ultrasonographer. 


\begin{tabular}{|c|c|c|c|c|c|}
\hline & $\begin{array}{l}\text { Intervention group (n } \\
=106) \\
\text { n (\%; } 95 \% \mathrm{Cl})\end{array}$ & $\begin{array}{l}\text { Control group }(n= \\
105) \\
n(\% ; 95 \% \mathrm{Cl})\end{array}$ & $\begin{array}{l}P \\
\text { value }\end{array}$ & $\begin{array}{l}\text { Absolute effect } \\
\%(95 \% \mathrm{Cl})\end{array}$ & $\begin{array}{l}\text { Relative effect } \\
(95 \% \mathrm{Cl})\end{array}$ \\
\hline \multirow{3}{*}{$\begin{array}{l}\text { Supplementary } \\
\text { diagnostic tests } \\
\text { ordered within } 4 \mathrm{~h} \\
\text { from admission } \\
\text { to the ED }\end{array}$} & 34 (32.1\%; 23.3-41.8) & $10(9.5 \% ; 4.7-16.8)$ & 0.0001 & \multirow{2}{*}{$\begin{array}{l}22.6 \%(12.0- \\
33.1)\end{array}$} & \multirow{2}{*}{$\begin{array}{l}3.37(1.76- \\
6.46)\end{array}$} \\
\hline & 85 (80.2\%; 71.3-87.2) & 90 (85.7\%; 77.5-91.8) & 0.29 & & \\
\hline & $10(9.4 \% ; 4.6-16.7)$ & 9 (8.6\%; 4.0-15.6) & 0.83 & $\begin{array}{l}-5.5 \%(-15.6- \\
4.6)\end{array}$ & $\begin{array}{l}0.94(0.83- \\
1.06)\end{array}$ \\
\hline \multirow{2}{*}{$\begin{array}{l}\text { Ultrasound of the } \\
\text { lungs }{ }^{c}\end{array}$} & 0 (0\%; 0-3.4¥) & 0 (0\%; 0-3.5¥) & - & $0.9 \%(-6.9-8.6)$ & \multirow{2}{*}{$\begin{array}{l}1.10(0.47- \\
2.60)\end{array}$} \\
\hline & $1(0.9 \% ; 0.0-5.1)$ & $2(1.9 \% ; 2.3-6.7)$ & 0.56 & 0 & \\
\hline $\begin{array}{l}\text { X-ray of the } \\
\text { thorax }\end{array}$ & 15 (14.2.\%; 8.1-22.3) & 19 (18.1\%; 11.3-26.8) & 0.44 & $-1.0 \%(-4.2-2.2)$ & \multirow{4}{*}{$\begin{array}{l}0.50(0.05- \\
5.58)\end{array}$} \\
\hline \multirow[t]{3}{*}{ CT of the thorax } & $2(1.90 \% ; 0.2-6.6)$ & 0 (0\%; 0-3.5¥) & 0.16 & $\begin{array}{l}-3.9 \%(-13.9- \\
6.0)\end{array}$ & \\
\hline & & & & $1.9 \%(-0.7-4.5)$ & \\
\hline & & & & $\begin{array}{l}11.2 \%(1.2- \\
21.2)\end{array}$ & \\
\hline MR of the thorax & 24 (22.6\%; 15.1-31.8) & 12 (11.4\%; 6.0-19.1) & 0.02 & & $\begin{array}{l}0.78(0.42- \\
1.45)\end{array}$ \\
\hline $\begin{array}{l}\text { Diagnostic } \\
\text { centesis }^{b}\end{array}$ & & & & & - \\
\hline $\begin{array}{l}\text { Echocardiography } \\
\text { by cardiologist }\end{array}$ & & & & & $\begin{array}{l}1.98(1.05- \\
3.75)\end{array}$ \\
\hline \multicolumn{6}{|l|}{$\begin{array}{l}\text { Ultrasound of the } \\
\text { deep veins }{ }^{d}\end{array}$} \\
\hline \multicolumn{6}{|l|}{$\begin{array}{l}\text { Other diagnostic } \\
\text { tests }\end{array}$} \\
\hline $\begin{array}{l}\text { Time spent in the } \\
\text { ED (hours) } \\
<11-23-45-8 \\
9-2425-48>48\end{array}$ & $\begin{array}{l}24(22.6 \% ; 15.6-31.7) \\
20(18.9 \% ; 12.4-27.6) \\
23(21.7 \% ; 14.8-30.7) \\
15(14.2 \% ; 8.6-22.3) \\
10(9.4 \% ; 5.1-16.8) 8 \\
(7.6 \% ; 3.8-14.5) 6 \\
(5.7 \% ; 2.5-12-2)\end{array}$ & $\begin{array}{l}28(26.7 \% ; 19.0-36.1) \\
24(22.9 \% ; 15.7-32.0) \\
18(17.1 \% ; 11.0-25.7) \\
17(16.2 \% ; 10.2-24.7) \\
5(4.8 \% ; 2.0-11.1) 5 \\
(4.8 \% ; 2.0-11.1) 9 \\
(8.6 \% ; 4.5-15.8)\end{array}$ & $\begin{array}{l}0.50 \\
0.48 \\
0.40 \\
0.68 \\
0.19 \\
0.40 \\
0.60\end{array}$ & $\begin{array}{l}-4.0 \%(-15.6- \\
7.6)-4.0 \% \\
(-14.9-7.0) \\
4.6 \%(-6.1- \\
15.2)-2.0 \% \\
(-11.7-7.6) \\
4.7 \%(-2.2- \\
11.6) 2.8 \% \\
(-3.7-9.3)-2.0 \% \\
(-8.7-4.8)\end{array}$ & $\begin{array}{l}0.85(0.53- \\
1.36) 0.83 \\
(0.49-1.40) 1.3 \\
(0.73-2.20) \\
0.87(0.46- \\
1.66) 1.98 \\
(0.70-5.60) \\
1.58(0.54- \\
4.69) 0.74 \\
(0.27-2.07)\end{array}$ \\
\hline
\end{tabular}

Abbreviations: $\mathrm{n}$ (number of patients). NIV (Non-invasive ventilation). CPAP (Continuous positive airway pressure). ED (emergency department). ICU (intensive care unit). $¥$ One-sided $97.5 \%$ confidence interval. aAccording to local treatment guidelines for the diseases in question. b Pleura-. thoraco-. cardio-centesis. c Performed by a radiologist or ultrasonographer certified in lung ultrasound. $d$ Performed by radiologist or certified ultrasonographer. 


\begin{tabular}{|c|c|c|c|c|c|}
\hline & $\begin{array}{l}\text { Intervention group (n } \\
=106) \\
\text { n (\%; } 95 \% \mathrm{Cl})\end{array}$ & $\begin{array}{l}\text { Control group }(n= \\
105) \\
n(\% ; 95 \% \mathrm{Cl})\end{array}$ & $\begin{array}{l}P \\
\text { value }\end{array}$ & $\begin{array}{l}\text { Absolute effect } \\
\%(95 \% \mathrm{Cl})\end{array}$ & $\begin{array}{l}\text { Relative effect } \\
(95 \% \mathrm{Cl})\end{array}$ \\
\hline $\begin{array}{l}\text { Time spent in } \\
\text { hospital (days) } \\
<11234-7>7\end{array}$ & $\begin{array}{l}42(39.6 \% ; 25.838 .4) \\
14(13.2 \% ; 12.1-22.3) \\
8(7.6 \% ; 5.0-12.6) 11 \\
(10.4 \% ; 5.7-13.7) 16 \\
(15.1 \% ; 14.2-24.9) 15 \\
(14.2 \% ; 11.3-21.2)\end{array}$ & $\begin{array}{l}25(23.8 \% ; 16.5-33.0) \\
21(20.0 \% ; 13.3-28.9) \\
9(8.6 \% ; 4.5-15.8) 8 \\
(7.6 \% ; 3.8-14.6) 24 \\
(22.9 \% ; 15.7-32.0) 18 \\
(17.4 \% ; 11.0-25.7)\end{array}$ & $\begin{array}{l}0.01 \\
0.18 \\
0.78 \\
0.48 \\
0.15 \\
0.55\end{array}$ & $\begin{array}{l}15.8 \%(3.4- \\
28.2)-6.8 \% \\
(-16.8-3.2) \\
-1.0 \%(-8.4-6.3) \\
2.8 \%(-5.0- \\
10.5)-7.8 \% \\
(-18.3-2.8) \\
-3.0 \%(-12.8- \\
6.8)\end{array}$ & $\begin{array}{l}1.66(1.10- \\
2.52) 0.66 \\
(0.36-1.23) \\
0.88(0.35- \\
2.19) 1.36 \\
(0.57-3.25) \\
0.66(0.37- \\
1.17) 0.83 \\
(0.44-1.55)\end{array}$ \\
\hline \multirow{2}{*}{$\begin{array}{l}\text { Itinerary: After } \\
\text { discharge from } \\
\text { the ED patients } \\
\text { were: }\end{array}$} & 0 (0\%; 0-3.4¥) & $1(1 \% ; 0.1-6.6)$ & 0.31 & $-1.0 \%(-0.3-0.0)$ & 0 \\
\hline & 52 (49.0\%; 39.5-58.6) & $52(49.5 \% ; 39.9-59.1)$ & 0.95 & $\begin{array}{l}-0.5 \%(-14.0- \\
13.0)\end{array}$ & $\begin{array}{l}0.99(0.75- \\
1.30)\end{array}$ \\
\hline $\begin{array}{l}\text { Transferred to the } \\
\text { ICU }\end{array}$ & 54 (50.9\%; 41.4-60.5) & 52 (49.5\%; 39.9-59.1) & 0.84 & $\begin{array}{l}-1.42 \%(-12.1- \\
14.9)\end{array}$ & $\begin{array}{l}1.03(0.79- \\
1.35)\end{array}$ \\
\hline \multicolumn{6}{|l|}{$\begin{array}{l}\text { Transferred to a } \\
\text { hospital ward }\end{array}$} \\
\hline \multicolumn{6}{|l|}{ Sent home } \\
\hline & 23 (21.7\%; 14.8-30.7) & $23(21.9 \% ; 14.9-31.0)$ & 0.97 & $\begin{array}{l}-0.26 \%(-11.3- \\
10.9)\end{array}$ & $\begin{array}{l}0.99(0.59- \\
1.65)\end{array}$ \\
\hline \multicolumn{6}{|l|}{$\begin{array}{l}\text { Patients } \\
\text { readmitted } \leq 30 \\
\text { days from } \\
\text { discharge }\end{array}$} \\
\hline $\begin{array}{l}\text { Mortality } \\
\text { In hospital }\end{array}$ & $\begin{array}{l}2(1.9 \% ; 0.2-6.6) 2 \\
(1.9 \% ; 0.2-6.6)\end{array}$ & $\begin{array}{l}4(3.8 \% ; 1.0-9.5) 7 \\
(6.7 \% ; 2.7-13.3)\end{array}$ & $\begin{array}{l}0.40 \\
0.09\end{array}$ & $\begin{array}{l}-1.9 \%(-6.4-2.6) \\
-4.8 \%(-10.2- \\
0.6)\end{array}$ & $\begin{array}{l}0.49(0.92- \\
2.64) 0.28 \\
(0.06-1.33)\end{array}$ \\
\hline \multicolumn{6}{|l|}{30 day mortality } \\
\hline $\begin{array}{l}\text { Abbreviations: } \mathrm{n} \text { ( } \\
\text { (emergency depart } \\
\text { treatment guidelin } \\
\text { ultrasonographer }\end{array}$ & $\begin{array}{l}\text { lber of patients). NIV ( } \\
\text { int). ICU (intensive care } \\
\text { or the diseases in ques } \\
\text { ified in lung ultrasound }\end{array}$ & $\begin{array}{l}\text {-invasive ventilation). C } \\
\text { it). } ¥ \text { One-sided } 97.5 \% \text { c } \\
\text { n. b Pleura-. thoraco-. ca } \\
\text { Performed by radiologis }\end{array}$ & $\begin{array}{l}\text { P (Cor } \\
\text { fidenc } \\
\text { lio-cen } \\
\text { or certi }\end{array}$ & $\begin{array}{l}\text { uous positive airn } \\
\text { terval. aAccording } \\
\text { s. c Performed by } \\
\text { ultrasonographe }\end{array}$ & $\begin{array}{l}\text { ay pressure). ED } \\
\text { to local } \\
\text { a radiologist or }\end{array}$ \\
\hline
\end{tabular}

In the intervention group, a significantly increased proportion of patients had supplementary diagnostic tests performed within the first $4 \mathrm{~h}$, and the most frequent diagnostic test was F-us of the lungs performed by a physician specialized in lung ultrasound; intervention $n=34(32 \cdot 1 \% ; 23 \cdot 3-41 \cdot 8)$, control $n=10(9 \cdot 5 \% ; 4 \cdot 7-16 \cdot 8)(p=0 \cdot 0001)$.

Moreover, a significantly increased proportion of patients in the intervention group spent less than 1 day in hospital, $n=42$ $(39.6 \% ; 25.8-38.4)$ compared to the control group $n=25(23.8 \% ; 16.5-33.0)(p=0.01)$ clarified by an absolute increase of $15.8 \%$. Aside from that we found no significant changes in the proportion of time spent in the ED or in hospital, in the patients' itinerary after discharge from the ED or in the proportion of readmissions and mortality (Table 4).

The diagnostic accuracy of the $4 \mathrm{~h}$ presumptive diagnoses compared to final diagnoses are listed in Table 2 and added contingency tables in Appendix 5. For 'Other diagnoses' than final diagnoses, see Appendix 6. Causes of death in patients who died within 30 days from admission are summarized in Appendix 7. For per protocol analysis, see Appendix 8.

No adverse events related to the F-us were observed.

\section{Discussion}


In this trial we found that adding F-us to standard clinical assessment in adult patients with acute signs of respiratory failure led to a non-significant, absolute increase of $2 \cdot 1 \%(p=0.71)$ in the proportion of patients with correct diagnoses $4 \mathrm{~h}$ after admission to the ED. We observed a significant, absolute increase of $13.5 \%(p=0.03)$ in the proportion of patients with appropriate treatment prescribed within $4 \mathrm{~h}$ from admission and of patients who stayed less than 1 day in hospital (Table 4).

To our knowledge, no similar randomized multicenter trial has been performed in adult ED patients admitted with signs of acute respiratory failure looking at both the diagnostic accuracy and the proportion of correct treatment prescribed and length of stay when adding F-us to standard clinical assessment.

Apart from a slight imbalance in the distribution of gender, the demographic and clinical characteristics were well matched and in combination with the vital signs and medical history upon admission, our patient population was comparable to similar studies. ${ }^{11,24}$

The high $4 \mathrm{~h}$ diagnostic accuracy found in the control group is surprising when compared to a similar single center trial, which reported an absolute increase of correct diagnoses of $24 \cdot 3 \%$ (from $63 \cdot 7 \%$ to $88.0 \%, 95 \% \mathrm{Cl} 15 \cdot 0-33 \cdot 1$ ) and of appropriate treatment prescribed of $21 \cdot 2 \%$ (from $56 \cdot 7-78 \%, 95 \% \mathrm{Cl} 10 \cdot 8-30 \cdot 9$ ) at $4 \mathrm{~h}$ after the patients admission ( $\mathrm{p}<$ 0.0001). They added cardiopulmonary F-us to standard clinical assessment performed by a single physician specialized in point-of care ultrasound. ${ }^{11}$ However, this does not explain the high $4 \mathrm{~h}$ diagnostic accuracy we found in both groups nor the significant increase of 'correct treatment prescribed' that we found in the intervention group. ${ }^{11}$

Instead, the high diagnostic accuracy at $4 \mathrm{~h}$ might be explained by the augmented focus on getting more specialist doctors in front in the first critical hours from the patients' admission to the ED which may have increased the diagnostic accuracy in general.

The significant increase of $13.5 \%$ in the proportion of patients in the intervention group who, at $4 \mathrm{~h}$ from admission, had an appropriate treatment prescribed despite an insignificant difference in diagnostic accuracy was similar to the single center study by Laursen et al. who found an absolute increase of $21 \cdot 2 \%$ (Table 4$){ }^{11}$

Laursen et al. found that the diagnostic tests prescribed within the first $4 \mathrm{~h}$ in the intervention group had a higher proportion of tests in which the suspected diagnoses were confirmed, but that the proportion of diagnostic tests prescribed evened out when compared throughout the entire hospital stay. ${ }^{11}$ These results indicate that the implementation of F-us leads to faster execution of diagnostic tests earlier in the hospital stay to confirm or reject a presumptive diagnosis and could be a contributory cause to the significant increase in the proportion of correctly prescribed treatment at $4 \mathrm{~h}$ in the intervention group. Finally, combined with the significant increase in the proportion of intervention patients who spent less than 1 day in hospital, these results indicate that the F-us has a positive impact on several clinical outcome parameters.

Apart from the above mentioned, we found no difference in the patients' itinerary, time spent in hospital or in the rate of readmissions or mortality as found by Laursen et al. ${ }^{11}$ The length of time spent in the ED or in hospital is often fairly short hence, larger studies are needed to assess whether F-us has a benefit on these parameters.

Finally, in the control group, the investigator revealed the F-us findings to the EP in two patients and in four patients the investigator became the treating physician within $6 \mathrm{~h}$ from the patients admission (Fig. 1).

In our study population we find a low number of severely ill patients (Table 1) and surprisingly few with pulmonary edema, pulmonary embolism and acute myocardial infarction despite a greater proportion of patients with systolic heart failure than found in similar trials. ${ }^{11,24,25}$ We cannot rule out that a selection bias has taken place where severely ill patients have been deselected due to the more cumbersome process of including these patients in a study trial. 
We may have reduced the number of final diagnoses of pulmonary edema (Table 2) as the investigators' F-us finding of pulmonary edema did not overrule a negative chest x-ray in the diagnostic criteria despite that F-us of the lungs has shown higher diagnostic accuracy. And as we did not implement pro-brain natriuretic peptide (BNP) in the diagnostic criteria, we may have reduced the number of final diagnoses of cardiac pathology. ${ }^{9}$ The proportion of patients with pulmonary embolism or AMI might be low due to the Danish emergency department setting that bypasses the ED and directs these patients straight to the department of cardiology or to outpatient clinics and because the prevalence of cardiopulmonary diseases is known to vary across studies despite similar inclusion criteria (Table 2). ${ }^{112426}$ Hence, we find no reason to believe that the manner of evaluating the final diagnoses plays a significant role in the results as it has been tested in similar studies. ${ }^{11,27,28}$

This study indicates that cardiopulmonary F-us is in the hands of non-specialist sonographers can impact patient treatment, underlined by studies of steep learning curves which demonstrates that novice sonographers can find pathology comparable to gold standards. ${ }^{13,14}$ The pragmatic multicenter trial design increases the generalizability of the results.

We limited the subjective bias from the blinded audit of the final diagnoses by predefined diagnostic criteria based on internationally accepted guidelines. However, the kappa agreement between the two auditors was only weak to moderate according to Cohen's guidelines, probably because the auditors had to agree on all diagnoses to obtain agreement or because the kappa statistic values tend to underestimate the agreement in situations with high inter-observer agreement (Appendix 4). However, the inter-observer variability reflects daily clinical dilemmas where a final clear diagnosis is not always possible to establish.

The study has limitations. Firstly, we never reached sample size (inclusion: 220 versus sample size: 288) which might have caused the lack of statistically significant results. Second, enrolment only took place when the investigators were present in the ED which might have caused bias. Third, the broad inclusion criteria prompted the inclusion of a large number of patients with diseases others than cardiopulmonary. Compared to other studies we ended up with several patients who had pathology that the F-us has no diagnostic impact on which may have caused a reduction in the absolute effect of our outcome parameters. ${ }^{11}$ Fourth, our diagnostic criteria may have been too strict as we omitted from the BTS criteria indicating that pneumonia could be diagnosed if there is "no other explanation for the illness, which is treated as community acquired pneumonia (CAP) with antibiotics" (Appendix 2) and from the investigators positive F-us findings of pneumonia, despite lung F-us has proven to be superior to chest x-ray in diagnosing pneumonia: This is emphasized by a higher proportion of diagnoses of pneumonia at $4 \mathrm{~h}$ than at final diagnoses in both groups and a lack of increase in the diagnostic accuracy (Table 3). ${ }^{82}$ Fifth, the general absence of a CT-thorax or complete echocardiography in the assessment of the final diagnoses is a weakness in the standard criteria of the final cardiopulmonary diagnoses and may have reduced the amount of significant findings. But, as it applies to all included patients we believe it to be of less importance.

Our study was not designed robust enough to enlighten the actual impact that F-us might have on acute as well as on hard clinical outcomes as patient morbidity, mortality and time spent in hospital. Larger studies are needed to specify the recommendations and the selection of patients whom would benefit the most from this diagnostic test as it has substantial healthcare and socioeconomic potential.

\section{Conclusion}

F-us in patients admitted to the ED with acute signs of respiratory failure in combination with standard clinical examination did not increase in the proportion of patients with correct diagnoses at $4 \mathrm{~h}$ after admission. But it was superior to standard clinical examination alone, as it increased the proportion of patients, who had supplementary diagnostic tests and correct treatment prescribed within $4 \mathrm{~h}$ from admission and who spent less than 1 day in hospital.

Page 19/25 


\section{List Of Abbreviations}

Cl Confidence interval

COPD Chronic obstructive pulmonary disease

CT Computerized tomography

ED Emergency department

EP Emergency physician

F-us Focused ultrasound

n Number of patients

$4 \mathrm{~h} 4$ hours

\section{Declarations}

\section{Ethics approval and consent to participate}

The trial was performed in accordance with the Helsinki II Declaration and approved by the Danish Data Protection Agency (identification number 13/12076) and the Committee on Biomedical Research Ethics for the Region of Southern Denmark (identification number S-20150090). The trial is registered with ClinicalTrials.gov, number NCT 02550184. The study protocol has previously been published. ${ }^{15}$ All patients provided signed informed consent prior to participation.

\section{Consent for publication}

No individual person's data are presented in this manuscript.

\section{Availability of data and material}

The datasets analyzed during the current study are available from the corresponding author on reasonable request.

\section{Competing interests}

M. Riishede is a member of the committee on ultrasound in the Danish Society for Emergency Medicine. MR has worked as an instructor on ultrasound courses for the Usabcd.org, the University of Aarhus and the University of Southern Denmark, Odense, Denmark. SECMA Medical Innovation, Denmark, lend ultrasound machine to MR and assisted with operator expertise for the introduction and instructions of the investigators

N. Jacobsen: Dr. Jacobsen reports personal fees from The Region of Southern Denmark and Tryg Foundation for working as an instructor on a focused lung ultrasound course for paramedics, outside the submitted work.

A.T. Lassen is funded by an unrestricted grant from the philantropic fund, Tryg Foundation, given to the University at Southern Denmark.

C.B. Laursen: Has received personal fee for giving lectures on an ultrasound course organized by GE Healthcare.

K.N. Jeschke, P.I. Pietersen and G. Baatrup have no conflicts of interests to declare.

\section{Funding}

Page 20/25 
The University of Southern Denmark, Odense University Hospital, Region of Southern Denmark.

\section{Authors' contributions}

Eligibility for authorship is gained according to the Vancouver guidelines.

M. Riishede led and contributed the conception and design of the study and the investigators. Conducted the inclusion as project and data manager and participated in the acquisition of data. MR performed the analysis and interpretation of data and drafted the submitted article.

ATL: contributed to the conception and design of the study, the interpretation of data and the draft of the submitted article.

G. Baatrup: contributed to the drafting of the submitted article.

P.I. Pietersen performed the audit of baseline characteristics and final diagnoses.I

N. Jacobsen performed the audit of the final diagnoses.

K.N. Jeschke contributed to the acquisition of data.

C.B. Laursen contributed to the conception and design of the study, interpretation of data and the draft of the submitted article.

All authors read and approved the final version of the submitted manuscript.

\section{Acknowledgements}

This study constituted a part of the corresponding authors PhD study and was thereby funded by research grants from the PhD-School and the Department of Clinical Research at the University of Southern Denmark - Odense, Denmark, the Department of Emergency medicine (FAM), the Department of Surgery and The Institute of Regional Health Research (IRS), Odense University Hospital - Svendborg, Odense, Denmark, and the Region of Southern Denmark.

We are deeply grateful to all patients, managers and staff in the participating EDs as well as to the investigators for their invaluable support and efforts to make this study possible. Likewise, we are very grateful to $\mathrm{H}$. Ruby and A. M. Bove (SECMA Medical Innovation, Denmark) who equipped the project manager with ultrasound equipment and operator expertise for the introduction and instructions of the investigators.

A great thank you to I. Petersen and L. Søgaard (OPEN, Odense University Hospital, Odense, Denmark) for statistical advice and support, and to N. I. Agerbek (Center of Clinical Epidemiology, Odense University Hospital, Odense, Denmark) for managing data in the National Patient Register platform (The Danish Health Data Authority).

\section{Authors' information}

Trial registration: Link to full trial; https://clinicaltrials.gov/, number NCT 02550184. Registered 10 June 2015.

\section{References}

1. Niska R, Bhuiya F and Xu J. National Hospital Ambulatory Medical Care Survey: 2007 emergency department summary. National health statistics reports 2010: 1-31. 2010/08/24.

2. Martindale JL, Wakai A, Collins SP, et al. Diagnosing Acute Heart Failure in the Emergency Department: A Systematic Review and Meta-analysis. Academic emergency medicine : official journal of the Society for Academic Emergency Medicine 2015 2016/02/26. DOI: 10.1111/acem.12878.

Page $21 / 25$ 
3. Hale ZE, Singhal A and Hsia RY. Causes of Shortness of Breath in the Acute Patient: A National Study. Academic emergency medicine : official journal of the Society for Academic Emergency Medicine 2018 2018/05/09. DOI: 10.1111/acem.13448.

4. Lindskou TA, Pilgaard L, Sovso MB, et al. Symptom, diagnosis and mortality among respiratory emergency medical service patients. PloS one 2019; 14: e0213145. 2019/03/01. DOI: 10.1371/journal.pone.0213145.

5. Zieleskiewicz L, Cornesse A, Hammad E, et al. Implementation of lung ultrasound in polyvalent intensive care unit: Impact on irradiation and medical cost. Anaesthesia, critical care \& pain medicine 2015; 34: 41-44. 2015/04/02. DOI: 10.1016/j.accpm.2015.01.002.

6. Laursen CB, Sloth E, Lassen AT, et al. Does point-of-care ultrasonography cause discomfort in patients admitted with respiratory symptoms? Scandinavian journal of trauma, resuscitation and emergency medicine 2015; 23: 46. 2015/06/14. DOI: 10.1186/s13049-015-0127-x.

7. Lichtenstein DA and Meziere GA. Relevance of lung ultrasound in the diagnosis of acute respiratory failure: the BLUE protocol. Chest 2008; 134: 117-125. 2008/04/12. DOI: 10.1378/chest.07-2800.

8. Parlamento S, Copetti R and Di Bartolomeo S. Evaluation of lung ultrasound for the diagnosis of pneumonia in the ED. The American journal of emergency medicine 2009; 27: 379-384. 2009/06/27. DOI: 10.1016/j.ajem.2008.03.009.

9. Wooten WM, Shaffer LET and Hamilton LA. Bedside Ultrasound Versus Chest Radiography for Detection of Pulmonary Edema: A Prospective Cohort Study. Journal of ultrasound in medicine : official journal of the American Institute of Ultrasound in Medicine 2019; 38: 967-973. 2018/10/04. DOI: 10.1002/jum.14781.

10. Bataille B, Riu B, Ferre F, et al. Integrated use of bedside lung ultrasound and echocardiography in acute respiratory failure: a prospective observational study in ICU. Chest 2014; 146: 1586-1593. 2014/08/22. DOI: 10.1378/chest.140681.

11. Laursen CB, Sloth E, Lassen AT, et al. Point-of-care ultrasonography in patients admitted with respiratory symptoms: a single-blind, randomized controlled trial. The Lancet Respiratory medicine 2014; 2: 638-646. 2014/07/08. DOI: 10.1016/s2213-2600(14)70135-3.

12. Laursen CB, Sloth E, Lambrechtsen J, et al. Focused sonography of the heart, lungs, and deep veins identifies missed life-threatening conditions in admitted patients with acute respiratory symptoms. Chest 2013; 144: 1868-1875. 2013/08/21. DOI: 10.1378/chest.13-0882.

13. Frederiksen CA, Juhl-Olsen $\mathrm{P}$, Andersen $\mathrm{NH}$, et al. Assessment of cardiac pathology by point-of-care ultrasonography performed by a novice examiner is comparable to the gold standard. Scandinavian journal of trauma, resuscitation and emergency medicine 2013; 21: 87. 2013/12/18. DOI: 10.1186/1757-7241-21-87.

14. Chiem AT, Chan $\mathrm{CH}$, Ander DS, et al. Comparison of expert and novice sonographers' performance in focused lung ultrasonography in dyspnea (FLUID) to diagnose patients with acute heart failure syndrome. Academic emergency medicine : official journal of the Society for Academic Emergency Medicine 2015; 22: 564-573. 2015/04/24. DOI: 10.1111/acem.12651.

15. Riishede M, Laursen CB, Teglbjaerg LS, et al. Focused ultrasound examination of the chest on patients admitted with acute signs of respiratory problems: a study protocol for a pragmatic randomized controlled multicentre trial. BMJ open 2016; 6: e012367. 2016/10/16. DOI: 10.1136/bmjopen-2016-012367. 
16. RedCap OPEN (Research Electronic Data Capture, Open Patient data Explorative Network), Department of Clinical Research, University of Southern Denmark. (Assessed October 22, 2019), (accessed October 22 2019).

17. Volpicelli G, Mussa A, Garofalo G, et al. Bedside lung ultrasound in the assessment of alveolar-interstitial syndrome. The American journal of emergency medicine 2006; 24: 689-696. 2006/09/21. DOI: 10.1016/j.ajem.2006.02.013.

18. Lichtenstein D. General Ultrasound in the Critically III . Berlin Heidelberg, Germany: Springer-Verlag 2007.

19. Via G, Hussain A, Wells $M$, et al. International evidence-based recommendations for focused cardiac ultrasound. Journal of the American Society of Echocardiography : official publication of the American Society of Echocardiography 2014; 27: 683.e681-683.e633. 2014/06/22. DOI: 10.1016/j.echo.2014.05.001.

20. Foss KT, Subhi Y, Aagaard R, et al. Developing an emergency ultrasound app - a collaborative project between clinicians from different universities. Scandinavian journal of trauma, resuscitation and emergency medicine 2015; 23: 47. 2015/06/21. DOI: 10.1186/s13049-015-0130-2.

21. National Report on Danish Emergency departments - 2016. The Danish Ministry of Health. https://www.regioner.dk/media/3084/statusrapport-om-akutmodtagelserne.pdf. Assessed January 31 2019. The Danish Ministry of Health. 2016.

22. Sealed envelope. (https://www.sealedenvelope.com/power/binary-superiority/) Assessed April 142015

23. Jakobsen JC, Gluud C, Wetterslev J, et al. When and how should multiple imputation be used for handling missing data in randomized clinical trials - a practical guide with flowcharts. BMC Med Res Methodol 2017; 17: 162. 2017/12/07. DOI: 10.1186/s12874-017-0442-1.

24. Ray P, Birolleau S, Lefort Y, et al. Acute respiratory failure in the elderly: etiology, emergency diagnosis and prognosis. Critical care (London, England) 2006; 10: R82. 2006/05/26. DOI: 10.1186/cc4926.

25. Sovso MB, Hermansen SB, Faerk E, et al. Diagnosis and mortality of emergency department patients in the North Denmark region. BMC health services research 2018; 18: 548. 2018/07/14. DOI: 10.1186/s12913-018-3361-x.

26. Staub LJ, Mazzali Biscaro RR, Kaszubowski E, et al. Lung Ultrasound for the Emergency Diagnosis of Pneumonia, Acute Heart Failure, and Exacerbations of Chronic Obstructive Pulmonary Disease/Asthma in Adults: A Systematic Review and Meta-analysis. The Journal of emergency medicine 2018 2018/10/14. DOI:

10.1016/j.jemermed.2018.09.009.

27. Pivetta E, Goffi A, Nazerian P, et al. Lung ultrasound integrated with clinical assessment for the diagnosis of acute decompensated heart failure in the emergency department: a randomized controlled trial. European journal of heart failure 2019 2019/01/29. DOI: 10.1002/ejhf.1379.

28. Riishede M, Laursen CB, Teglbjaerg LS, et al. Diagnostic value of whole-body-focused ultrasonography in high-acuity patients in the emergency department: a prospective single-center cross-sectional study. Ultrasound J 2019; 11: 11. 2019/07/31. DOI: 10.1186/s13089-019-0126-7.

29. Haggag YI, Mashhour K, Ahmed K, et al. Effectiveness of Lung Ultrasound in Comparison with Chest X-Ray in Diagnosis of Lung Consolidation. Open Access Maced J Med Sci 2019; 7: 2457-2461. 2019/11/02. DOI: 10.3889/oamjms.2019.669.

30. Touw HR, Parlevliet KL, Beerepoot M, et al. Lung ultrasound compared with chest X-ray in diagnosing postoperative pulmonary complications following cardiothoracic surgery: a prospective observational study. Anaesthesia 2018; 73 : 946-954. 2018/03/13. DOI: 10.1111/anae.14243.

Page 23/25 


\section{Figures}

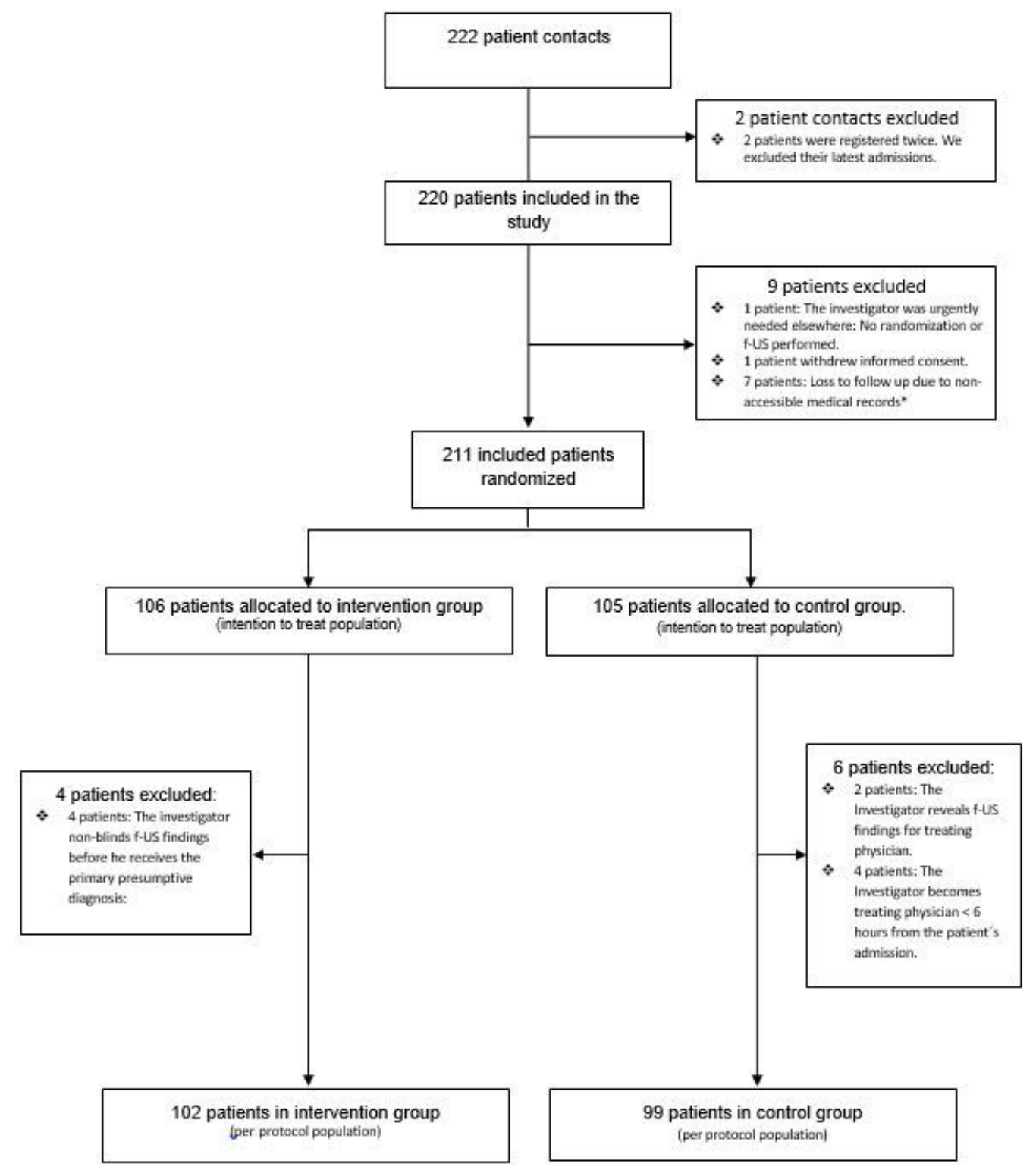

\section{Figure 1}

Trial profile *The 7 randomized patients we lost due to loss of follow up are assessed to be missing at random and were excluded from all analyses as all outcomes were to be calculated by statistic approximation. They were lost as follows: 4 were caused by investigators in two hospitals who withdrew their consent to participation due to too busy working hours in the ED, in 2 the medical records were lost in the transition to a new electronic medical record system, and in 1 an unidentifiable personal ID number was written in the database.

\section{Supplementary Files}

This is a list of supplementary files associated with this preprint. Click to download.

- CONSORT2010Checklistfilledin.pdf

- Additionalfile11DiagnosticcriteriaFus.docx 
- Additionalfile2Diagnosticcriteriaofaudit.docx

- Additionalfile3Ultrasoundfindings.docx

- Additionalfile4Interraterreliabilityofaudit.docx

- Additionalfile5aDiagnosticaccuracy4hInt.docx

- Additionalfile5bDiagnosticaccuracy4hctrl.docx

- Additionalfile6aOtherdiagnosesint.docx

- Additionalfile6bOtherdiagnosesctrl.docx

- Additionalfile7Causeofdeath.docx

- Additionalfile8PPanalysestable14.docx 